\title{
Efficient MAC Adaptive Protocol on Wireless Sensor Network
}

\author{
Adim Firmansah ${ }^{1}$, Aripriharta $^{2}$, Sujito ${ }^{2}$ \\ ${ }^{1}$ Graduate School, Faculty of Engineering, Universitas Negeri Malang, Semarang Street No. 5, Malang 65145, Indonesia \\ ${ }^{2}$ Electrical Engineering Department, Faculty of Engineering, Universitas Negeri Malang, Semarang Street No. 5, Malang 65145, \\ Indonesia
}

\section{ARTICLE INFO}

\section{Article history:}

Received October 10, 2021

Revised November 06, 2021

Accepted November 15, 2021

\section{Keywords:}

Sensor;

Network;

Lifetime;

Efficient;

Energy

\begin{abstract}
Wireless Sensor Networks (WSNs) have attracted a lot of attention from the research community and industry in recent years. WSNs maintenance associated with battery replacement can increase system operating costs, especially for wireless sensor networks located in hard-to-reach and dangerous places. In this study, an adaptive Medium Access Control (MAC) is proposed that can regulate the period of data acquisition and transmission. In contrast to conventional MAC, the applied adaptive MAC regulates the data transmission period based on the estimated energy use in the previous cycle. This study focuses on comparing energy efficiency between conventional and adaptive MAC. Energy usage information is retrieved directly on the sensor node. In star topology, the proposed MAC can increase the lifetime of the sensor network up to $6.67 \%$ in a star topology. In the hierarchical topology, the proposed MAC can increase network energy efficiency up to $9.17 \%$. The resulting increase in network throughput is $17.73 \%$ for the Star network and $33.81 \%$ for the Hierarchy network. The star topology without implementing adaptive MAC has the lowest throughput of $0.188 \mathrm{~kb} / \mathrm{s}$. The highest throughput is achieved by a hierarchical topology that applies MAC with a throughput of $2.157 \mathrm{~kb} / \mathrm{s}$.
\end{abstract}

This work is licensed under a Creative Commons Attribution-Share Alike 4.0

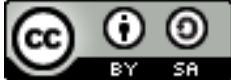

\section{Corresponding Author:}

Aripriharta, Electrical Engineering Department, Faculty of Engineering, Universitas Negeri Malang, Semarang Street No. 5, Malang 65145, Indonesia.

Email: aripriharta.ft@um.ac.id

\section{INTRODUCTION}

Wireless Sensor Networks or better known as WSNs, have attracted a lot of attention from the research community and industry in recent years. The reason is that WSN has a high potential to be implemented in various fields, including military operations, environmental monitoring, security systems, health care [1]-[3]. Sensor nodes can work independently and retrieve environmental data. Nodes can also communicate and exchange data with each other [4]. These features make WSN the right choice for many applications, especially applications in environments that are hazardous to human existence [5].

The main obstacle in WSN is battery life [6]-[8]. Lifetime refers to the maximum time all nodes in the network remain functional until one or more nodes do not work [9], [10]. Maintenance related to battery replacement can increase system operating costs, especially for wireless sensor networks located in hard-toreach and dangerous places [11], [12]. Therefore, many studies have focused on methods to increase the lifetime of WSN.

These methods are radio optimization [13], [14], data reduction [15], [16], routing protocol efficiency [17], [18], battery repletion [19], [20], and application of sleep-wake up schemes [21], [22]. The sleep-wakeup scheme includes duty cycling, passive wake-up radios, and topology control. Duty cycling aims to adjust node activity to save energy by setting the node into sleep mode. The node does not allow to transmit information to the surrounding nodes/sinks because they are inactive at the same time. Finally, the active and sleep periods are fixed to allow nodes to exchange data with each other. However, the Medium Access Control 
(MAC) protocol used in the sleep-wake up scheme affects network performance. When applying MAC with a low duty cycle, it will save a lot of energy, but the delay in communication and data acquisition increases significantly [23].

Conventional MAC sets a fixed period of active (data acquisition and transmission) and sleeps on each node (S-MAC) [24]. However, in reality, each node has a different active period when performing data acquisition and data transmission. Nodes located far from the sink will tend to require longer data transmission times than nodes located near the sink [25]-[28]. Setting the same active and sleep periods will make nodes located far from the sink more likely to die faster. Referring to the meaning of lifetime in WSN, conventional MAC is less efficient because each node consumes different energy in one active cycle.

\section{RELATED WORKS}

Various MAC developments have emerged, such as T-MAC [29], which increase energy efficiency. TMAC improves the low throughput and high latency of S-MAC leading to high energy consumption. T-MAC achieves better efficiency compared to S-MAC by reducing collisions and idly listening as the node returns to sleep mode if there is no activity during the active period.

Yang et al. [30] propose U-MAC, which is an improvement from T-MAC. U-MAC strikes a balance between energy and latency in WSN. The node uses a utility function which is the ratio of the actual two-way communication performed by the node to adjust its duty cycle across active periods. A node in idle listens to the state during its active period when getting utility function is low. U-MAC can save $32 \%$ of energy compared to S-MAC.

In the dynamic duty cycle control proposed by Wang et al. [31], the feedback controller manages the endto-end duty cycle and delay to achieve high energy efficiency. The duty cycle is controlled in proportion to the node's one-hop delay condition as well as the actual packet delay, which is calculated using a time code. If the sender node sends data and it is not received by the receiver node, the sender node will enter sleep mode and try to send data in the next cycle.

Byun \& Yu [32] propose an adaptive duty cycle control mechanism. The mechanism dynamically adjusts the sleep period of a node for the data queue length according to the threshold. The data queue size threshold is limited to a predefined value to save energy, achieve low end-to-end delay, and control duty cycles. The sleep period increases linearly as the queue length becomes smaller than the queue threshold. If the queue length becomes greater than the threshold, then the sleep interval is reduced.

ERI-MAC was proposed by Nguyen et al. [33], in which node receivers configure the wake-up period based on the energy harvested. The receiver must reach the ENO state for wake-up. The sender holds data in listening mode and waits for a beacon from the receiver. When the sender receives the beacon, the sender immediately sends the data.

The listening mode on ERI-MAC consumes a lot of energy, so Bouachir et al. [34] propose the EnergyAware MAC Protocol with Adaptive Individual Duty Cycle (EAMP-AIDC) for WSN equipped with energy harvesting features. Similar to ERI-MAC, EAMP-AIDC makes the node must be in the ENO state, where the node must harvest more energy than the energy needed to be active and transmit data. EAMP-AIDC uses the TDMA protocol to determine transmission times, thereby avoiding nodes in redundant listening mode.

\section{MOTIVATION AND CONTRIBUTION}

The adaptive MAC proposed in this study regulates the data transmission period on the node based on the estimated energy used by the node in the previous cycle. Energy estimation in the previous cycle is calculated based on the average energy when active and the length of time the node is active. In improving the accuracy of energy estimation, the calculation of energy estimation uses the ratio of the data acquisition and transmission period. Determination of node transmission tasks is carried out through cloud computing, and the results are stored in the cloud database.

Adaptive MAC differs from conventional MAC in that each node performs data acquisition and transmission when active. In contrast to the S-MAC, which delays the node data transmission task, the proposed adaptive MAC delays the node transmission task when the estimated energy used in the previous cycle exceeds the set threshold value. This also applies to T-MAC, which puts the node into sleep mode when there is no activity.

In contrast to the adaptive MAC protocol proposed by S. Yang et al. [30], Wang et al. [31], and Byun \& $\mathrm{Yu}$ [32], which is based on network latency, the proposed adaptive MAC is based on node energy consumption to increase energy efficiency. In contrast to ERIM-MAC [33] and EAMP-AIDC [34], which are aimed at nodes that have energy harvesting features, the proposed adaptive MAC can be applied to all nodes, including those that do not have energy harvesting features. This study focuses on comparing the energy efficiency between the proposed conventional and adaptive MAC. Energy usage information is retrieved directly on the sensor 
node. The energy usage parameters obtained are used in the MATLAB simulation to compare the energy efficiency between the two schemes.

\section{METHOD}

\subsection{System Design}

In this study, the wireless sensor network uses single-hop and multi-hop topologies. Each node transmits data to the nearest sink. The node uses a static addressing configuration for shortening the network authentication process time [35]. The node consists of a microcontroller and a sensor. In this study, several ESP8266 microcontrollers were used, which are low-power microcontrollers and already have Wi-Fi transceivers. In this study, the node uses a DHT11 sensor to retrieve data on the temperature and humidity of the surrounding environment. The sensor nodes are powered by an $1100 \mathrm{mAh}(1.2 \mathrm{~V})$ AAA battery connected in series. Fig. 1 shows a block diagram of the components that make up the node which includes sensors, microcontrollers, communication circuits, and power management circuits.

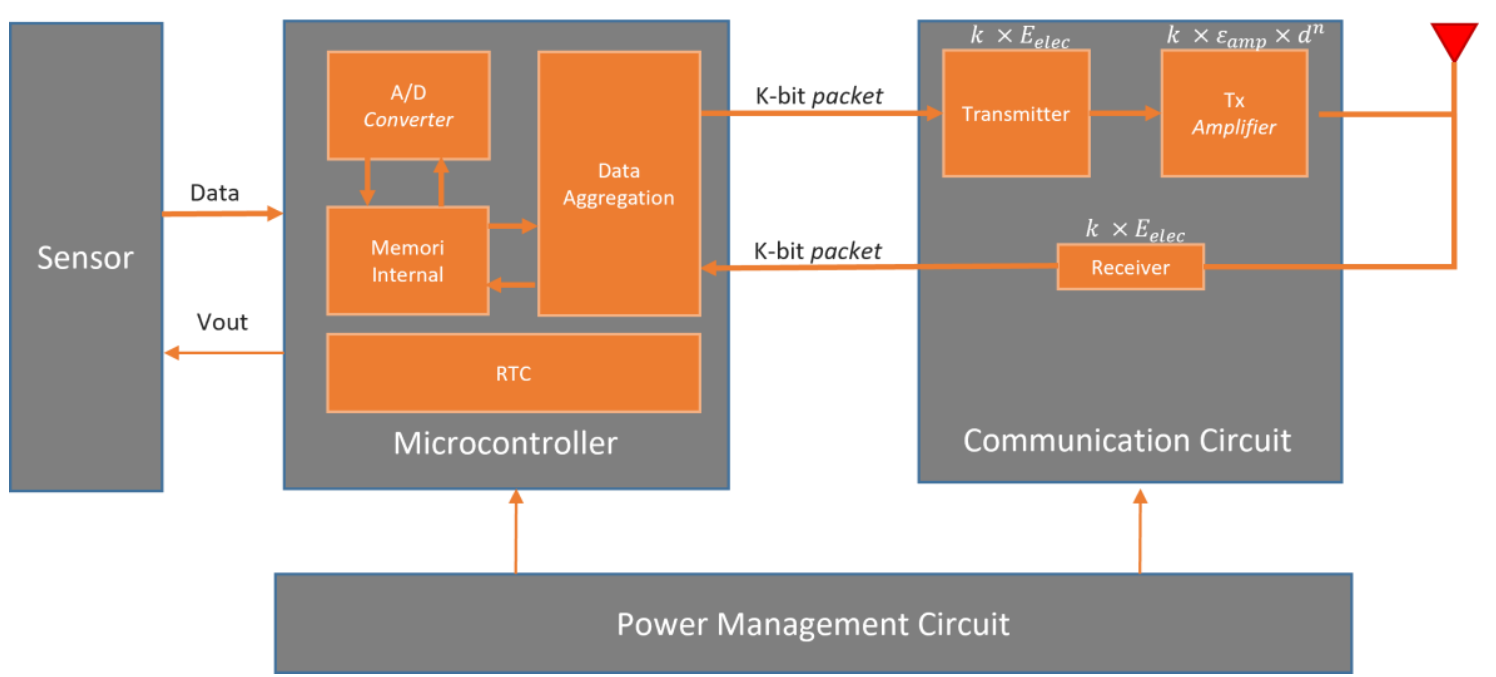

Fig. 1. Sensor node components

The sensor network and sensor nodes in this study are assumed to be as follows.

- Each sensor can measure environmental parameters and send that data to the sink or cluster head $(\mathrm{CH})$.

- The initial energy of each node is assumed to be the same.

- Each sensor wakes up from sleep then performs the process of data acquisition and data transmission. After data transmission is complete, it goes into sleep mode.

- When in sleep mode, only the Real-time Clock (RTC) circuit is active on the microcontroller, so there is still energy consumption for RTC performance. The sensor does not consume energy because it is connected to the output pin of the microcontroller.

- Each node has a fixed power transmission gain level.

- The sink is powered by an external power source.

- Data transmission between transmitter and receiver in the sensor network is assumed to always be successful.

- Each Node and $\mathrm{CH}$ are assumed to have a wake-up time from sleep mode at the same time to transmit data.

- Network lifetime is calculated based on the metric until one of the nodes dies (FND).

- The sink is placed in the middle of the area.

In this study, the energy consumed by the node to transmit data is calculated by considering the path loss between vacuum and multipath adjusted to the distance between the sender (transmitter) and the receiver (receiver). If the distance is smaller than the $d_{0}$ threshold, a vacuum model is used. The energy required to transmit packets per k-bit over a distance $d$ is shown in (1) [10].

$$
E_{T x}(k, d)= \begin{cases}k \times E_{\text {elec }}+k \times E_{f s} \times d^{2}, & \text { when } d \leq d_{0} \\ k \times E_{\text {elec }}+k \times E_{m p} \times d^{4}, & \text { when } d>d_{0}\end{cases}
$$

Where $E_{T x}$ is the transmission energy with $d$ as the transmission distance between transmitter and receiver, $E_{\text {elec }}$ is the energy lost for transmitting per bit of data from transmitter to receiver, $E_{f s}$ is the energy used to receive 
data. $E_{f s}$ and $E_{m p}$ depend on the transmitter gain level where $k$ is the transmitted data length, and $d_{0}$ is the transmission distance threshold calculated using (2). The energy consumed by the radio to receive data is assumed in (3).

$$
\begin{gathered}
d_{0}=\sqrt{\frac{E_{f s}}{E_{m p}}} \\
E_{R x}=k \times E_{\text {elec }}
\end{gathered}
$$

Each sensor node sends data to the sink in the star topology and through the $\mathrm{CH}$ intermediary in the hierarchical topology. The size of the packet sent is based on the $802.11 \mathrm{n}$ standard, where the data sent is accompanied by a packet header. Based on this standard, the preamble has a size of 10 bytes. In this study, the data sent by the node to the sink is in the form of sensor data, IP address, and active period. The data is sent in the form of a linked list with the \# character as the delimiter. After sending the data, the sensor node receives the data transmission index. The size of the data is based on the size of the file used to store the data on the computer. The size of these data is shown in Table 1.

Table 1. Data size

\begin{tabular}{clrr}
\hline \multirow{2}{*}{ No. Description } & \multicolumn{2}{c}{ Size } \\
\cline { 3 - 4 } & & byte & bit \\
\hline 1 & Sensor data & 6 & 48 \\
2 & IP data & 96 \\
3 & Active period & 4 & 32 \\
4 & Coordinate & 4 & 32 \\
5 & Transmission index & 1 & 8 \\
6 & Linked list & 25 & 200 \\
7 & Linked list with 2 sensor data & 32 & 256 \\
8 & Preamble & 10 & 80 \\
9 & Preamble + coordinate & 14 & 112 \\
10 & Preamble + Linked list & 35 & 280 \\
11 & Preamble + Linked list with 2 sensor data & 42 & 336 \\
12 & Preamble + transmission index & 11 & 88 \\
\hline
\end{tabular}

The energy consumption of the sensor node is based on data retrieval using the INA219 sensor. The results of the data collection show that the active period $\left(t_{n}\right)$ of the node ranges from $1950 \mathrm{~ms}$ to $3050 \mathrm{~ms}$ with energy consumption per second $\left(E_{c}\right)$ of $0.209 \mathrm{~J}$. Based on these data, the active period of nodes that do not transmit data is set at $1950 \mathrm{~ms}$, where nodes that transmit data have an active period of between $1950 \mathrm{~ms}$ to $3050 \mathrm{~ms}$. Therefore, in this study, sensor nodes that do not transmit data have an active period of $1950 \mathrm{~ms}$, while sensor nodes that transmit data have an active period of between $1950 \mathrm{~ms}$ to $3050 \mathrm{~ms}$.

When in sleep mode, the current consumption of the sensor node is based on the microcontroller datasheet, which is $20 \mu \mathrm{A}$. In this study, the duration of sleep $\left(t_{\text {sleep }}\right)$ for 15 seconds is used so that the sensor energy consumption for sleep $\left(E_{\text {sleep }}\right)$ for 15 seconds is $990 \mu \mathrm{J}$. The energy consumed by the sensor node in one cycle consisting of data acquisition and data transmission $\left(E_{c t r}\right)$ is defined in $(4)$, while the energy consumption of the sensor node in one cycle consists of data acquisition only $\left(E_{c n t}\right)$ is defined in (5). Nodes that have an active period below $3050 \mathrm{~ms}$ will add the remaining active period so that each node has the same wake-up time. Equation (6) shows the addition of the sleep node period so that each node has the same wake-up time where $t_{\max }=3050 \mathrm{~ms}$.

$$
\begin{gathered}
E_{c t r}=E_{T x}(k, d)+E_{R x}+\left(E_{c} \times t_{n}\right)+E_{\text {sleep }} \\
E_{\text {cnt }}=\left(E_{c} \times t_{n}\right)+E_{\text {sleep }} \\
t_{\text {sleep }}=\left(t_{\text {max }}-t_{n}\right)+15
\end{gathered}
$$

\subsection{Adaptive MAC}

The proposed adaptive MAC makes the data transmission stage did not perform every time the node is active. Nodes that receive instructions to delay sending data will send data in the next active cycle. Fig. 2 shows the data communication flow on the adaptive MAC. The node sends uptime $\left(t_{n}\right)$ and sensor monitoring data to 
the sink. After receiving the data, the sink responds by sending a data delivery indicator $\left(t_{i}\right)$ to the node. After receiving the value $t_{i}$, the node goes into sleep mode.

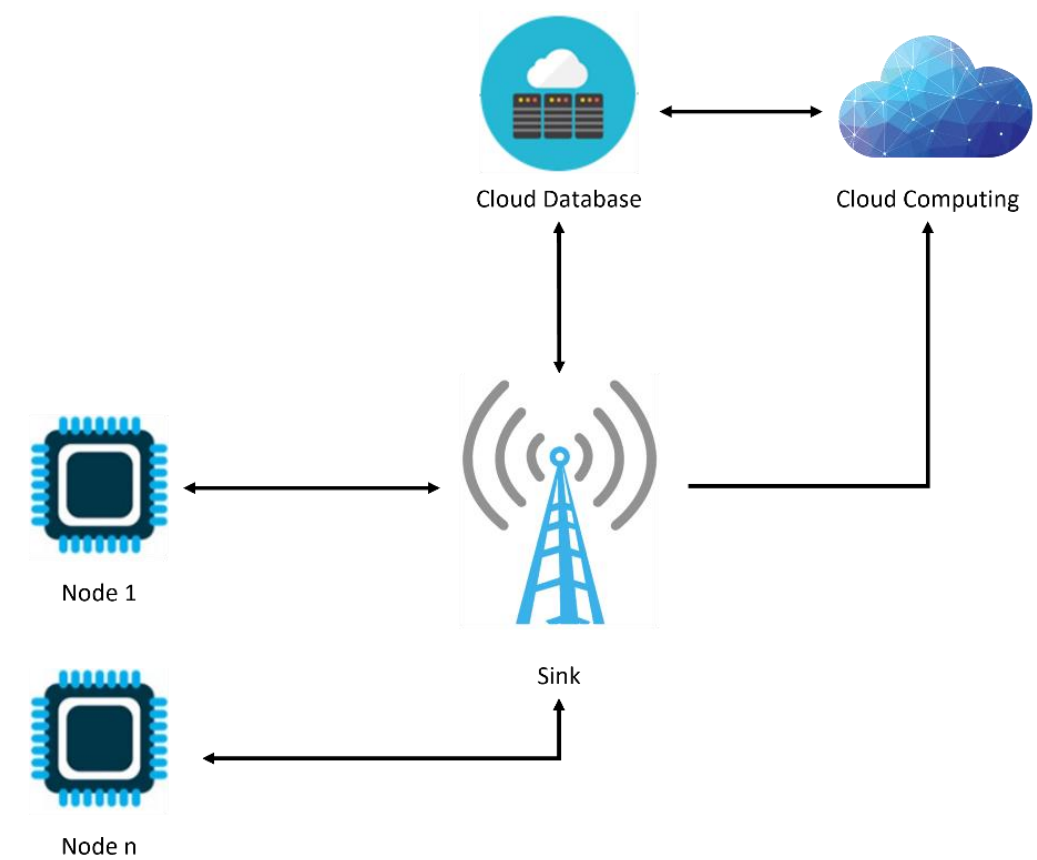

Fig. 2. Data communication

The sink adds data in the form of IP addresses to the data obtained from the node and passes it to the cloud computing API. Sink enters sleep mode after successfully sending data to the Application Programming Interface (API) of cloud computing or computing on local servers. The sink will wake up earlier from sleep mode and fetch the latest data on the cloud database. The data is in the form of IP nodes and $t_{i}$ of each node. When the node wakes up from sleep mode, it sends sensor data and $t_{n}$ to the sink. However, nodes that get a value of $t_{i}=0$ will only retrieve data and send the data in the next cycle. The cycle keeps repeating itself. Fig. 3 shows the system timing diagram.

\section{Node 1}

Node 2

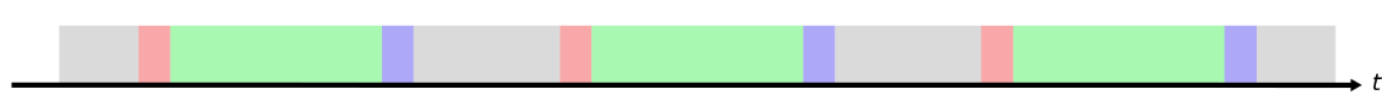

Sleep

Data Acquistiion (node)

Data transmision (node to sink)

Data retrieval (sink from cloud database)

Sink Stanby

Data transmission (sink to cloud computing)

Fig. 3. Timing diagram

On standby, the sink is ready to receive data from the node. In Fig. 3, node 1 gets a value of $t_{i}=0$ in the first cycle so that in the second cycle, it performs data acquisition without sending it. Data transmission to the sink is carried out by Node 1 in the third cycle. The pseudocode that describes the node workflow is shown in Pseudocode 1. 
After the node sends data to the sink, it goes into sleep mode after receiving the $t_{i}$ data. The sink will temporarily store the data until the sink's active time $\left(t_{a}\right)$ expires. After the sink's uptime expires, the sink sends all data retrieved from the node to the cloud database. The sink workflow is shown in Pseudocode 2.

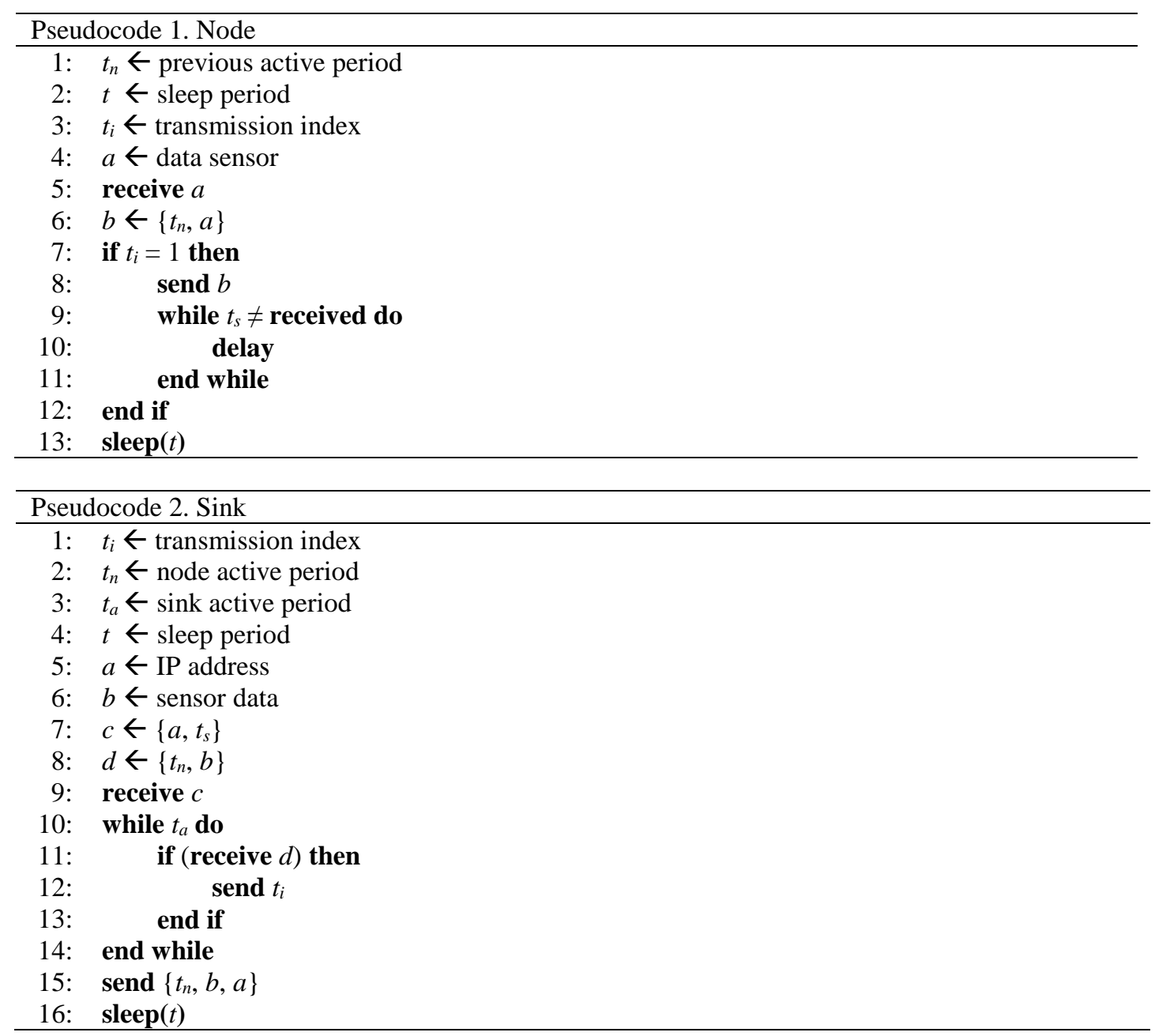

The data is sent by the sink to the cloud computing API and directly processed to determine the value of $t_{i}$. The value of $t_{i}$ is determined using Pseudocode 3 . The result of the calculation of the value of $t_{i}$ stored to accessed by the sink. The obtained $t_{i}$ value is stored in the cloud database for sink access when starting a new cycle. The value of $E_{c a}$ is the energy targeted for one cycle that is calculated using (7) as

$$
E_{c a}=E_{c} \times t_{n a} \text {. }
$$

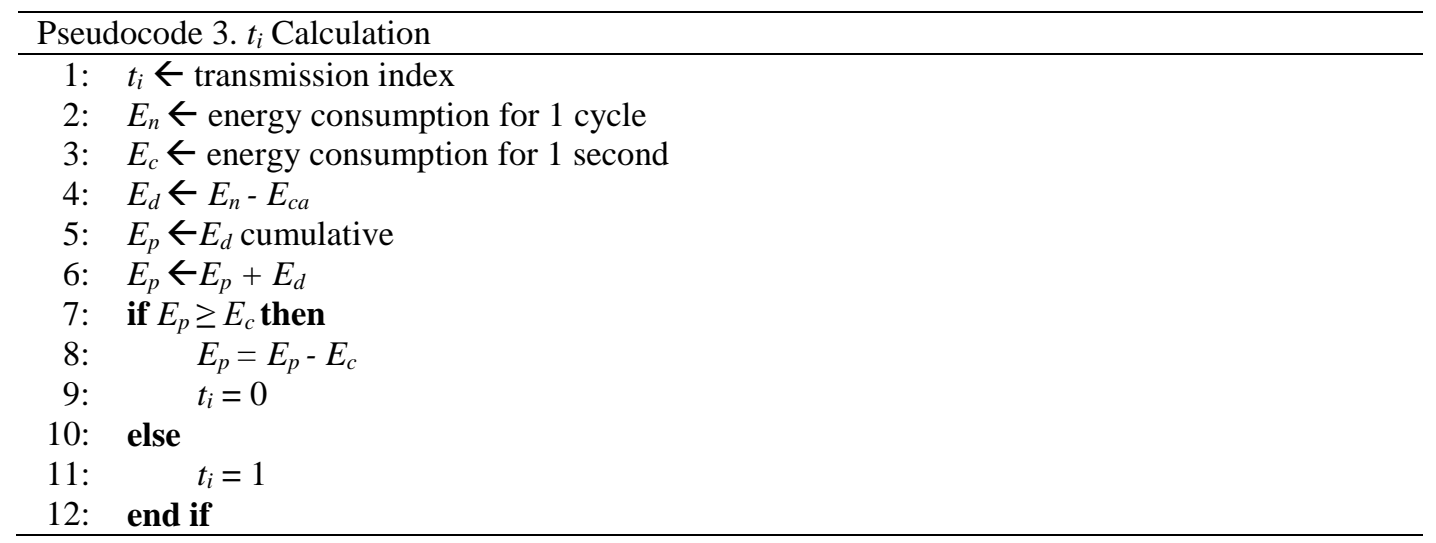


In this research, the value of $t_{n a}$ was used for 2.225 seconds, where the value was taken from the active period of the sensor node for the cycle, namely 1.95 - 3.05 seconds. If the value of $t_{n a}$ selected is getting smaller, then the energy savings made by the sensor node using the proposed MAC will be even greater. However, it causes sensor nodes to delay data transmission more often to save energy ( $\left.t_{i}=0\right)$. The value of $E_{c}$ is the energy consumed by the sensor node in one second (in the active cycle), which is $0.209 \mathrm{~J}$.

\subsection{Research Scenario}

In this study, data collection was carried out through a sensor network simulation using Matlab software. The simulation parameters used were obtained from the results of previous research on energy harvesting [12]. MAC simulations were performed using the same battery capacity. The simulation results taken are when the WSN system starts to work until one of the WSN nodes dies because it runs out of energy. The parameters used in the simulation are shown in Table 2.

Table 2. Simulation parameters

\begin{tabular}{ll}
\hline \multicolumn{1}{c}{ Parameter } & \multicolumn{1}{c}{ Value } \\
\hline Number of nodes & 100 \\
Initial energy & $100 \mathrm{~J}$ \\
Area & $100 \mathrm{~m} \times 100 \mathrm{~m}$ \\
Sleep period & $900 \mathrm{~s}$ \\
$E_{\text {elec }}$ & $50 \mathrm{~nJ} / \mathrm{bit}$ \\
$E_{f s}$ & $10 \mathrm{pJ} / \mathrm{bit} / \mathrm{m}^{2}$ \\
$E_{m p}$ & $0.0013 \mathrm{pJ} / \mathrm{bit} / \mathrm{m}^{4}$ \\
$d_{0}$ & $75 \mathrm{~m}$ \\
$E_{D A}$ & $5 \mathrm{~nJ} / \mathrm{bit} / \mathrm{signal}$ \\
$E_{c}$ & $0.209 \mathrm{~J}$ \\
$E_{c a}$ & $0.465 \mathrm{~J}$ \\
$E_{\text {sleep }}$ & $990 \mu \mathrm{J}$ \\
$t_{n}$ & $1.950 \mathrm{~s}-3.050 \mathrm{~s}$ \\
$t_{n a}$ & $2.225 \mathrm{~s}$ \\
\hline
\end{tabular}

The simulation is carried out on a star network structure, and a hierarchy where each network is divided into two, namely applying conventional MAC and adaptive MAC proposed in this study. In a star network topology, nodes send data directly to the sink. Nodes that are still alive are nodes that still have energy where the energy will decrease every round.

The hierarchical network structure used in the simulation applies the LEACH protocol to select the cluster head $(\mathrm{CH}) . \mathrm{CH}$ is selected based on the rotation of the round or sensor that has the most energy. The node sends data to the nearest $\mathrm{CH}$. Data aggregation is performed on the $\mathrm{CH}$. $\mathrm{CH}$ forwards the data to the sink. The node that has the closest distance to the sink (not included in any cluster) directly sends data to the sink.

Energy efficiency analysis is the stage of comparing node energy consumption when using conventional and adaptive MAC. At this stage, the causes of the difference in energy consumption between the two algorithms are analyzed. The analysis is focused on two main points, namely, the lifetime period of WSN and throughput. WSN lifetime is measured until one of the nodes in the network is out of energy. At this stage, the total energy remaining in each round/cycle is summed and displayed in graphical form. The simulation was carried out 20 times, and then the average lifetime value was taken in the four simulation scenarios. The four simulation scenarios are detailed as follows.

- Star topology (scenario 1)

- $\quad$ Star topology + MAC (scenario 2)

- $\quad$ Hierarchy Topology + LEACH (scenario 3)

- $\quad$ Hierarchy topologi + LEACH + MAC (scenario 4)

The throughput measurement was carried out using (8). Where tpr is the data sent $(\mathrm{kb})$, and $t$ is the time it takes to send the data (s). The value of $t p r$ consists of the amount of data sent by the sensor node to the sink (scenarios $1 \& 2$ ). In scenarios 3 and 4, tpr is the amount of data sent by the sensor node to the $\mathrm{CH}$ and the data sent by the $\mathrm{CH}$ to the sink. The amount of data sent by each node is $4 \mathrm{~kb}$ in each data transmission. The data is assumed to contain node active time, IP address, transmission index, and sensor data. Data transmission time 
is calculated based on the number of cycles. Each cycle has the targeted sensor node active time duration incremented by sleep period (6).

$$
\text { Throughput }=\frac{t p r}{t}
$$

\section{RESULTS AND DISCUSSION}

\subsection{Lifetime}

The comparison results of the sensor network lifetime when using the proposed algorithm on a star (single hop) topology are shown in Fig. 4. The experiment was carried out using 100 sensor nodes 20 times. When using a star topology network, the lifetime of the First Node Die (FND) sensor network reaches 187-189 cycles. Half Node Die (HND) was achieved in cycles 192-193, and Last Node Die (LND) was achieved in cycles 197198.

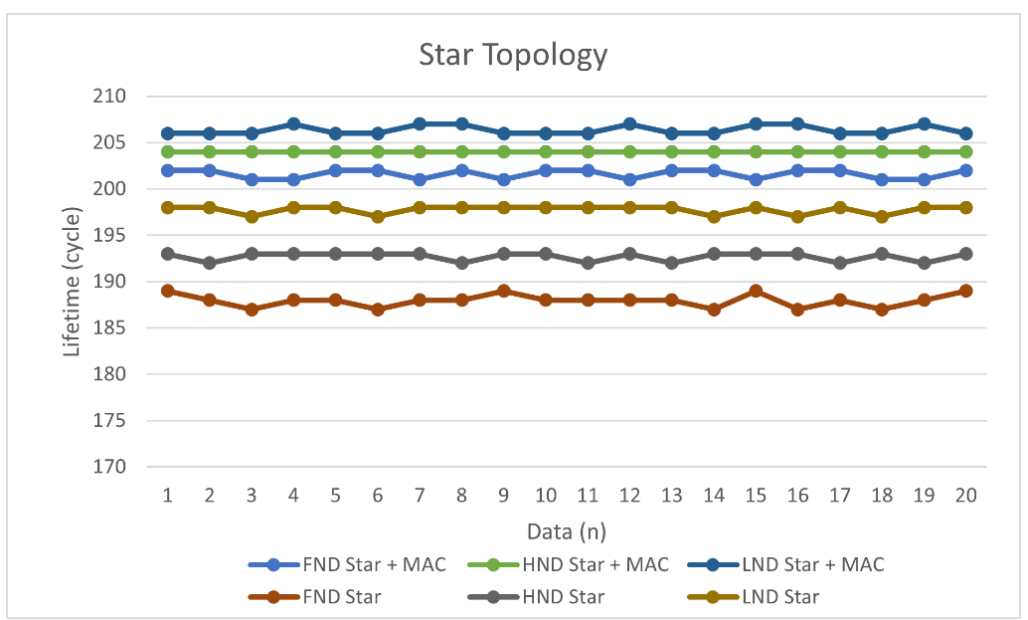

Fig. 4. Star topology lifetime

However, when the proposed MAC is implemented, it can increase the service life of the sensor network until the FND reaches 201-202 cycles. HND is achieved in cycle 204, and LND is achieved in cycle 206-207. In a star topology, the proposed MAC can increase the sensor network lifetime by up to $6.67 \%$ with an initial energy of each node of $100 \mathrm{~J}$.

Simulation on a hierarchical topology is carried out by combining the LEACH algorithm in determining the cluster combined with the proposed MAC to improve energy efficiency. The simulation results are shown in Fig. 5. The sensor network that uses a hierarchical topology by applying LEACH can reach a lifetime of up to 187-189 cycles (FND). This value is like the result of star topology without MAC application because most of the energy is spent by energy for sensor active time. HND was achieved in the 192-193 cycle, and LND was achieved in the 196-199 cycle.

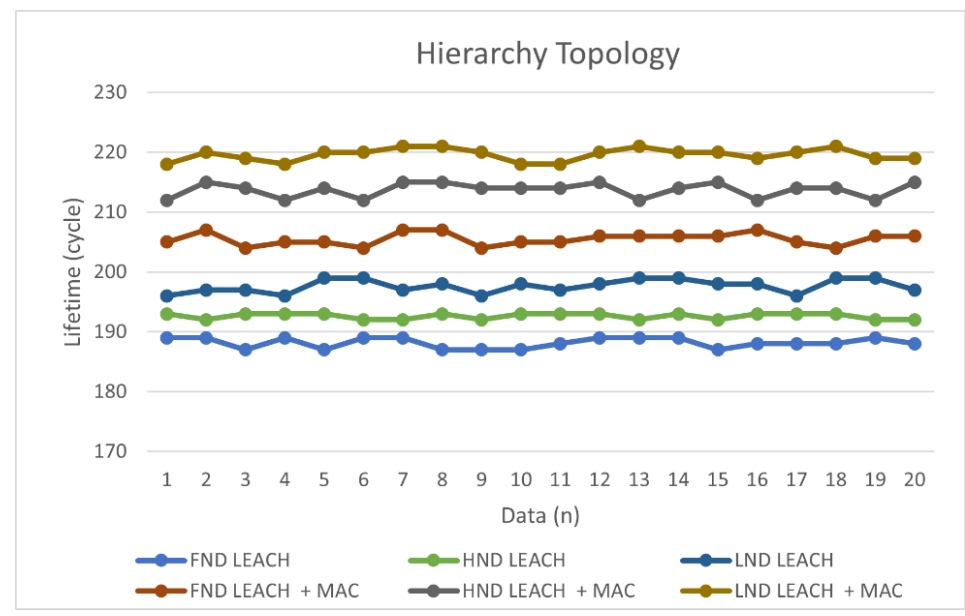

Fig. 5. Hierarchy topology lifetime 
When combining the proposed LEACH + MAC (scenario 4), the device lifetime increases to 204-207 cycles (FND). The combination of LEACH + MAC can achieve HND on the 212-215 cycle, and LND is achieved on the 219-221 cycle. In a hierarchical topology that uses the LEACH protocol for clustering, the proposed MAC can increase network energy efficiency up to $9.17 \%$. Based on the simulation results of scenarios 1-4, the applied algorithm can provide power savings in star and hierarchical topologies. However, energy efficiency is greater when applied to a hierarchical topology. A sample of the sensor network energy consumption in each of the simulated topologies is shown in Fig. 6, with initialization energy of 100J for each sensor node. The graph shows the total energy remaining in the network obtained by adding up the energy remaining at each sensor node. The energy is calculated at the end of each cycle.

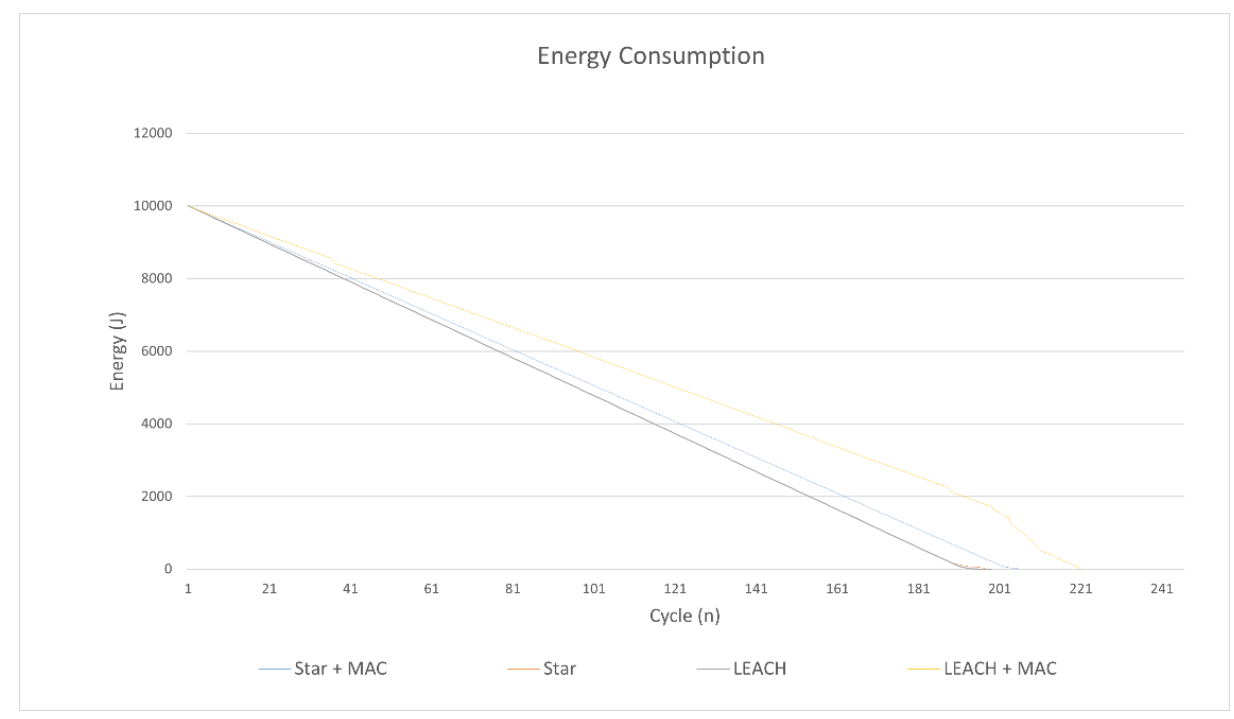

Fig. 6. Energy consumption

A comparison of the active nodes in each scenario is shown in Fig. 7. In star topology without using MAC, all sensors remain on until the 188th cycle. In the 189th cycle, there are only 98 sensors that still have energy remaining. In a star topology that implements an adaptive MAC, all sensor nodes still have energy up to the 202nd cycle, and 89 nodes remain in the 203rd cycle.

In a sensor network that uses a Star and Hierarchy topology without MAC implementation, all sensors are still active until the 188th cycle. When entering the 189th cycle, there are nodes that are running out of energy ( 2 nodes in Star and 1 node in Hierarchy). All nodes run out of energy on the 200th cycle. Topologies that apply MAC have a longer lifetime. In the Star + MAC topology scenario, all sensors are active up to cycle 202, with FND 11 sensors shutting down on cycle 203. All sensors run out of energy on cycle 207. While in Hierarchy + MAC topology scenario, all sensors are active until cycle 205 with 1 node off on cycle 206. All sensors run out of energy at cycle 219 .

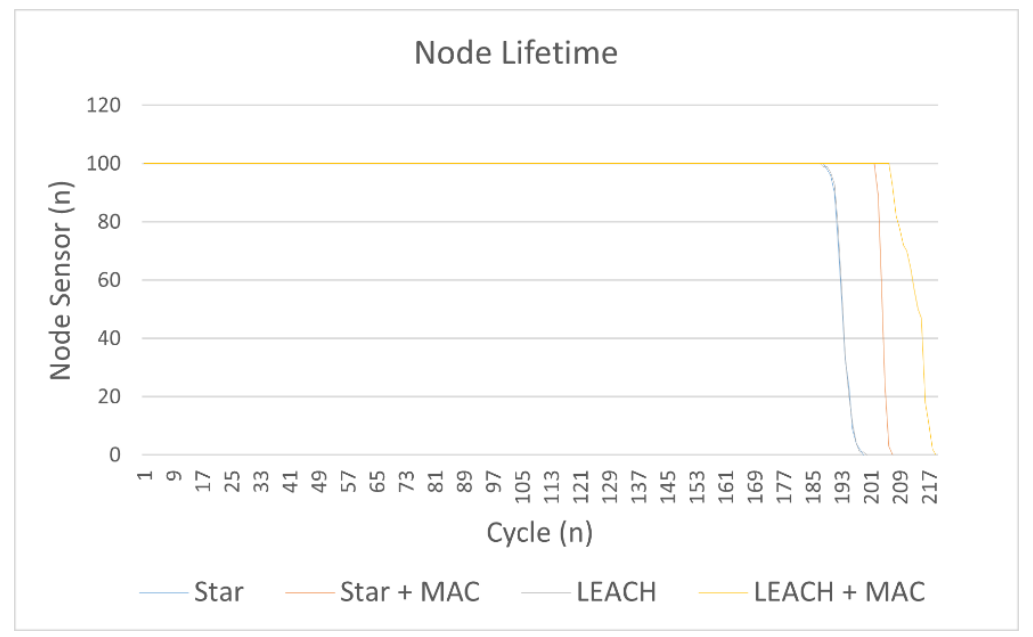

Fig. 7. Node lifetime 
The value of $E_{p}$ (the difference between $E_{n}$ and $E_{c a}$ ) in each cycle is shown in Fig. 8. If the value of $E_{p}$ is in the positive range, then in that cycle, more nodes spend energy above the average energy per targeted cycle. However, if the value of $E_{p}$ is in the negative range, then in that cycle, the sensor node consumes total energy below the average target energy. When the value of $E_{p}$ is high, it indicates that the sensor network needs energy saving, while the value of $E_{p}$ is low, it indicates that the cycle of the sensor network is consuming less energy than the target.

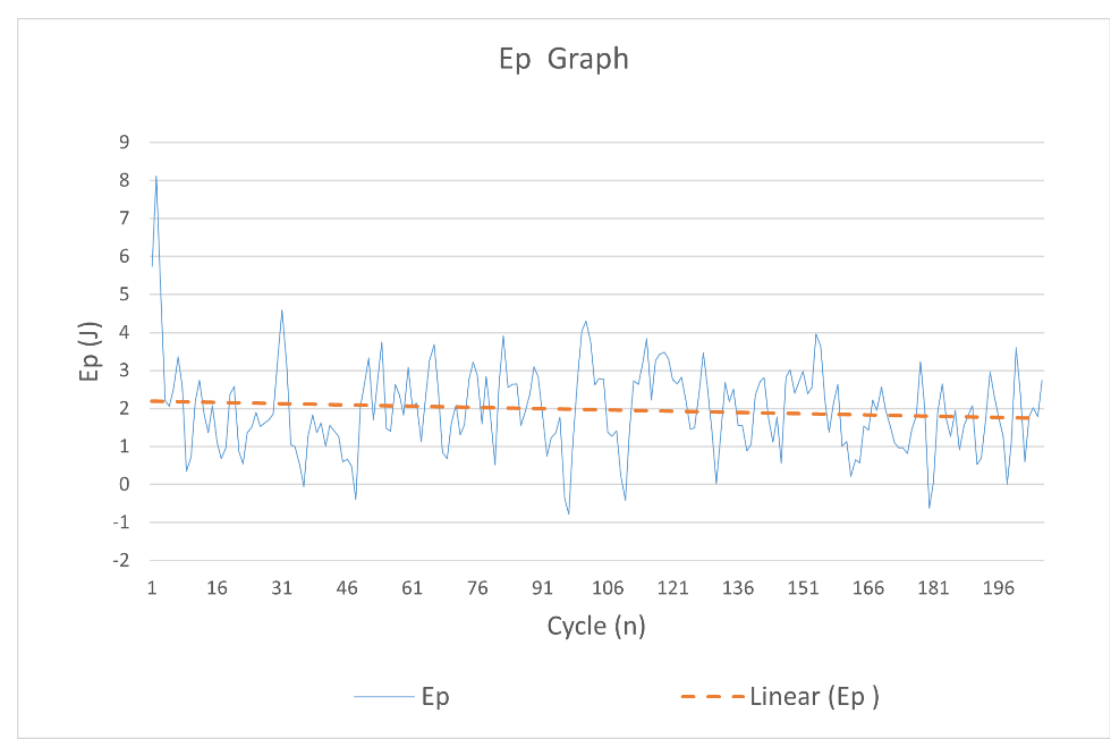

Fig. 8. $\boldsymbol{E}_{\boldsymbol{p}}$ graph

The transmission index in the network is shown in Fig. 9. In the first cycle, all sensor nodes transmit data to send data to the sink (Star + MAC) and to $\mathrm{CH}$ (Hierarchy + MAC). In the Star + MAC scenario, in one cycle, the average sensor node transmitting data is 86 sensor nodes. This value varies from 76 to 94 sensor nodes in each cycle. Meanwhile, in the Hierarchy + MAC scenario, the average sensor node that transmits data is 89 sensor nodes. This value varies from 81 to 98 sensor nodes in each cycle.

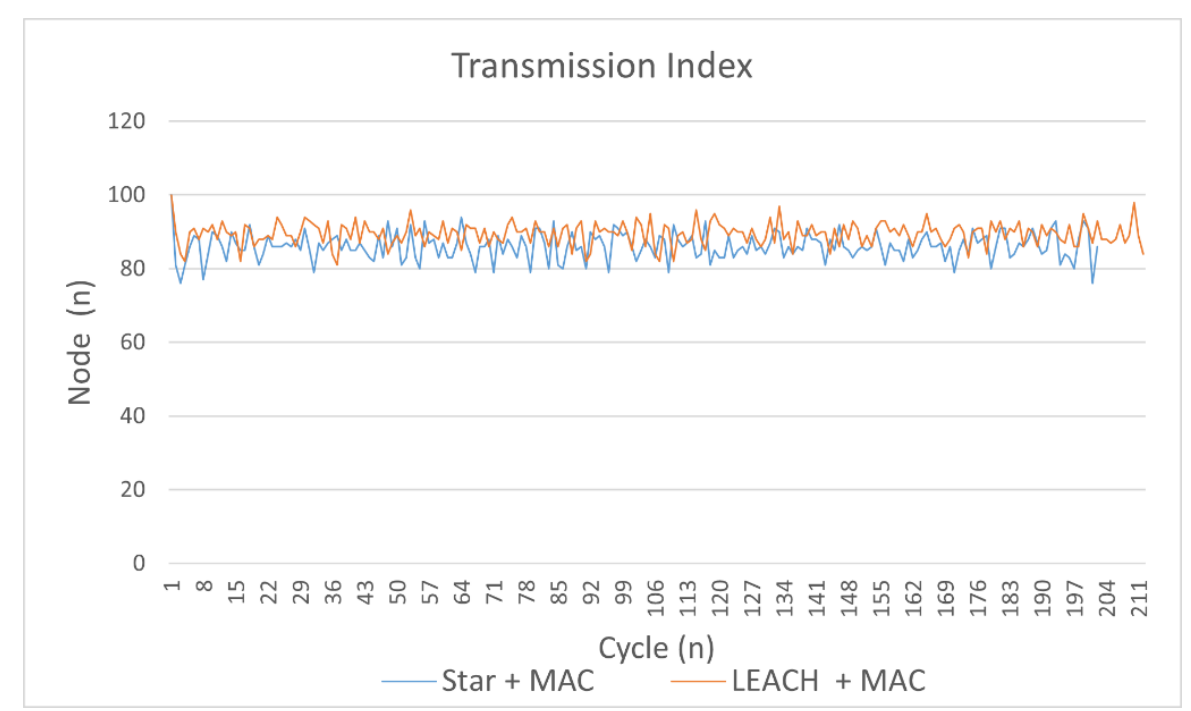

Fig. 9. Transmission index

The energy savings generated by the application of adaptive MAC are $6.67 \%$ in the star topology and $9.17 \%$ in the hierarchical topology. Table 3 shows the cycle increments when using the initial energy of $100 \mathrm{~J}$ and $13068 \mathrm{~J}$ with a commercially available $1100 \mathrm{mAh}$ battery. The table data is made with the assumption that the battery capacity can be used entirely by the sensor nodes. When using a $1100 \mathrm{mAh}$ battery, the adaptive MAC implemented in the star topology can increase the lifetime to 1699 cycles. In a hierarchical topology, the implemented adaptive MAC can increase the lifetime up to 2222 cycles. 
Table 3. Lifetime

\begin{tabular}{clrrrr}
\hline \multirow{2}{*}{ No } & \multirow{2}{*}{ Scenario } & \multicolumn{3}{c}{ Battery } & \multirow{2}{*}{ Lifetime (cycle) } \\
\cline { 3 - 5 } & Voltage (V) & Capacity (mAh) & Energy (J) & 189 \\
\hline 1 & Star & 3.3 & 8.418 & 100 & 202 \\
2 & Star + MAC & 3.3 & 8.418 & 100 & 189 \\
3 & Hierarchy & 3.3 & 8.418 & 100 & 206 \\
4 & Hierarchy + MAC & 3.3 & 8.418 & 100 & 24699 \\
5 & Star & 3.3 & 1100.000 & 13068 & 26397 \\
6 & Star + MAC & 3.3 & 1100.000 & 13068 & 24699 \\
7 & Hierarchy & 3.3 & 1100.000 & 13068 & 26920 \\
8 & Hierarchy + MAC & 3.3 & 1100.000 & 13068 & \\
\hline
\end{tabular}

\subsection{Throughput}

The throughput of the star topology sensor network and the hierarchy when performing the simulation is shown in Table 4. The star topology without MAC has the lowest throughput of $0.188 \mathrm{~kb} / \mathrm{s}$. This value is caused because the packet data is sent over a longer period when the node saves energy. The highest throughput is achieved by a hierarchical topology applying MAC with a throughput of $2.157 \mathrm{~kb} / \mathrm{s}$.

Table 4. Throughput

\begin{tabular}{clr}
\hline No & \multicolumn{1}{c}{ Scenario } & Throughput $(\mathbf{k b} / \mathbf{s})$ \\
\hline 1 & Star & 0.188 \\
2 & Star + MAC & 0.222 \\
3 & Hierarchy & 1.612 \\
4 & Hierarchy + MAC & 2.157 \\
\hline
\end{tabular}

Network transmitted data per cycle for each study scenario is shown in Fig. 10. On the Star network, the average amount of data transmitted in each cycle is $3.397 \mathrm{~kb}$. The Star + MAC network has a different amount of data sent in each cycle because not all sensor nodes send data with an average data transfer rate of $3.999 \mathrm{~kb}$ per cycle. Networks with Hierarchy and Hierarchy + MAC topologies have various amounts of data transmitted, which are higher in value than Star and Star + MAC networks. The Hierarchical Network has an average of $29.106 \mathrm{~kb}$ of data transmitted per cycle. The Hierarchy + MAC network has an average data transmitted of $38.95 \mathrm{~kb}$.

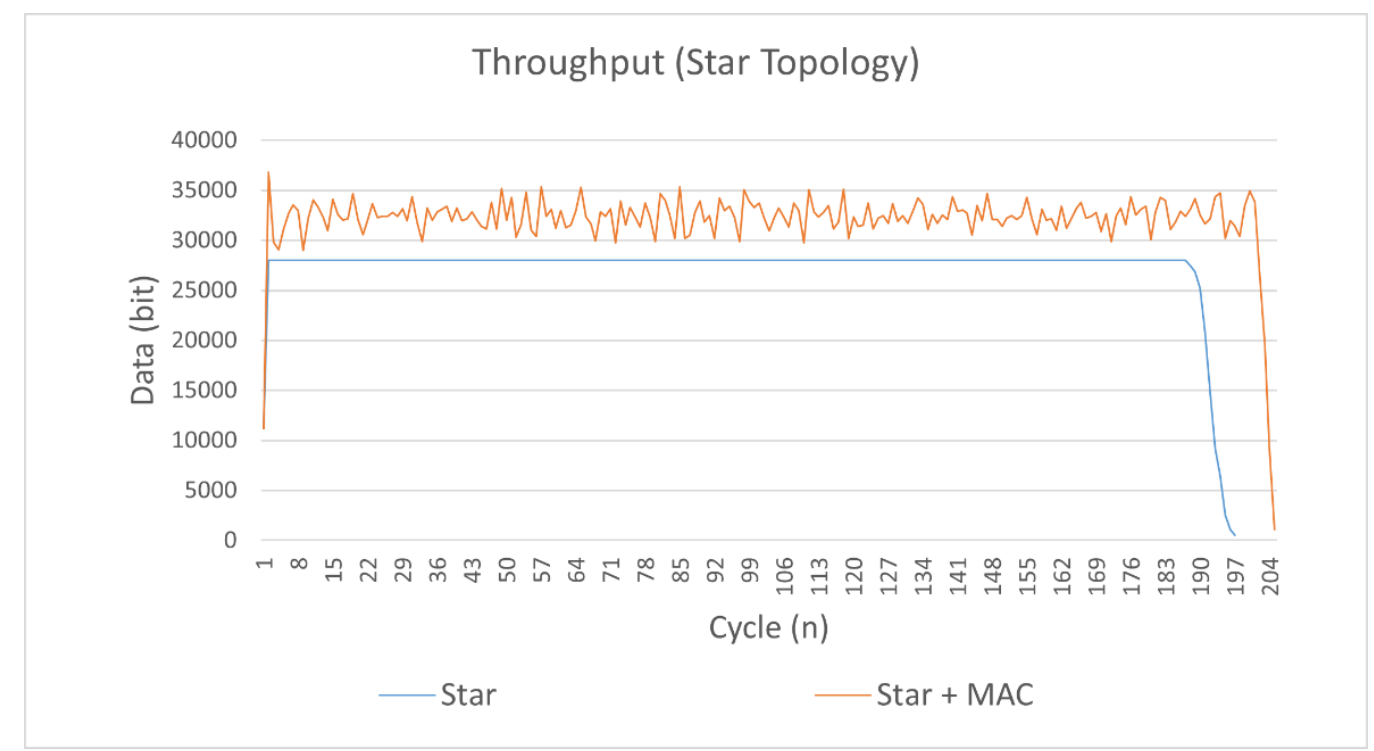

Fig. 10. Star throughput

The proposed MAC can increase network throughput. The resulting increase in network throughput is $17.73 \%$ for the Star network. Throughput in a hierarchical network is shown in Fig. 11. In a hierarchical network, the proposed MAC also increases throughput by $33.81 \%$. Although the proposed MAC allows not all nodes to be active in every cycle, the network throughput increases. This is caused by the transmission of index 
data transmission on the network. In the final cycles, the value of the transmitted data decreases as the number of sensor nodes runs out of energy.

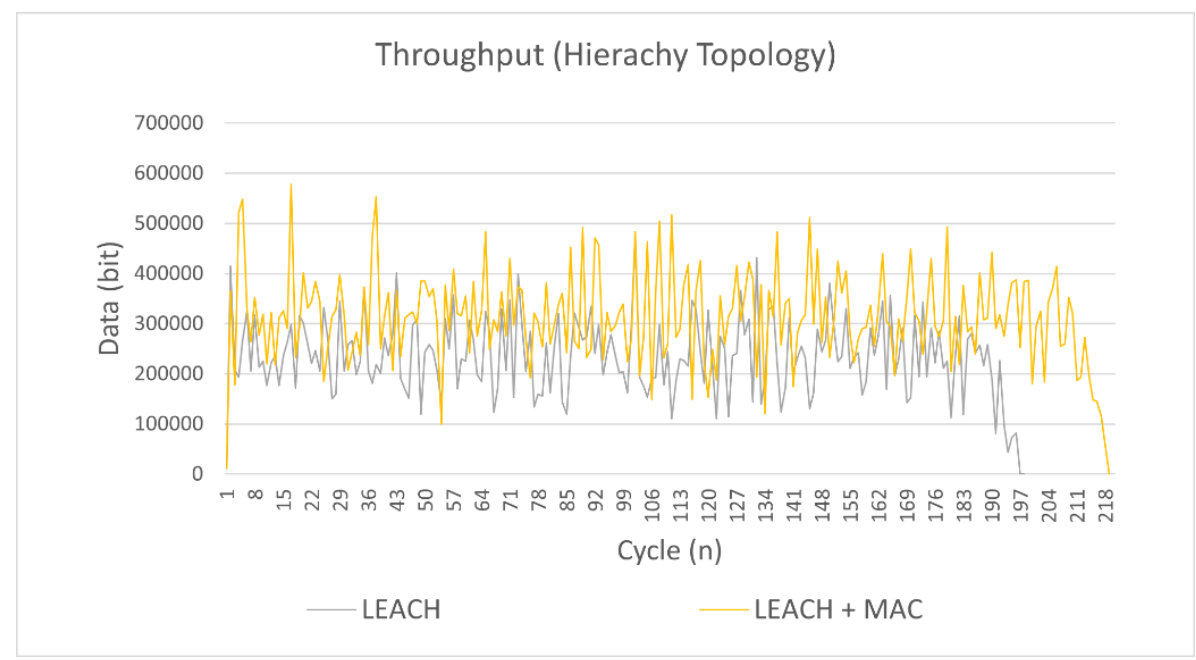

Fig. 11. Hierarchy throughput

\section{CONCLUSION}

Based on the results obtained from the research, it can be concluded that adaptive MAC has greater efficiency than conventional MAC, especially in the energy efficiency used to activate data transmission circuits. Adaptive MAC has a longer lifetime in both applied network topologies (star and hierarchy). Energy efficiency is improved by delaying the data transmission time according to the node's energy use in the previous cycle. Adaptive MAC can increase lifetime with the highest efficiency obtained when MAC is implemented in a sensor network with a hierarchical topology. When implemented in a sensor network, the proposed adaptive MAC can increase network throughput.

\section{Acknowledgments}

This work was supported by PNBP UM 2021 Under contract number 5.3.810/UN32.14.1/LT/2021, Universitas Negeri Malang, Indonesia.

\section{REFERENCES}

[1] I. Mathews, P. J. King, F. Stafford, and R. Frizzell, "Performance of III-V solar cells as indoor light energy harvesters," IEEE J. Photovoltaics, vol. 6, no. 1, pp. 230-235, 2016. https://doi.org/10.1109/JPHOTOV.2015.2487825

[2] Y. Liu, Q. Wu, T. Zhao, Y. Tie, F. Bai, and M. Jin, "An improved energy-efficient routing protocol for wireless sensor networks," Sensors (Switzerland), vol. 19, no. 20, pp. 1-20, 2019. https://doi.org/10.3390/s19204579

[3] J. V. Alamelu and A. Mythili, "Design of IoT based generic health care system," 2017 Int. Conf. Microelectron. Devices, Circuits Syst. ICMDCS 2017, vol. 2017-January, pp. 1-4, 2017. https://doi.org/10.1109/ICMDCS.2017.8211698

[4] R. P. Hudhajanto, N. Fahmi, E. Prayitno, and Rosmida, "Real-Time Monitoring for Environmental Through Wireless Sensor Network Technology,” Proc. 2018 Int. Conf. Appl. Eng. ICAE 2018, pp. 1-5, 2018. https://doi.org/10.1109/INCAE.2018.8579377

[5] R. Elhabyan, W. Shi, and M. St-Hilaire, "Coverage protocols for wireless sensor networks: Review and future directions," J. Commun. Networks, vol. 21, no. 1, pp. 45-60, 2019. https://doi.org/10.1109/JCN.2019.000005

[6] H. Yu and Q. Yue, "Indoor light energy harvesting system for energy-aware wireless sensor node," Energy Procedia, vol. 16, no. PART B, pp. 1027-1032, 2012. https://doi.org/10.1016/j.egypro.2012.01.164

[7] S. Nasr and M. Quwaider, "LEACH Protocol Enhancement for Increasing WSN Lifetime," 2020 11th Int. Conf. Inf. Commun. Syst. ICICS 2020, pp. 102-107, 2020. https://doi.org/10.1109/ICICS49469.2020.239542

[8] P. Poulose and P. Sreejaya, "Indoor Light Harvesting Using Dye Sensitized Solar Cell," 2018 Int. CET Conf. Control. Commun. Comput. IC4 2018, pp. 152-156, 2018. https://doi.org/10.1109/CETIC4.2018.8530924

[9] V. Kumar and A. Kumar, "Improving reporting delay and lifetime of a WSN using controlled mobile sinks," $J$. Ambient Intell. Humaniz. Comput., vol. 10, no. 4, pp. 1433-1441, 2019. https://doi.org/10.1007/s12652-018-0901-5

[10] Y. Zhou, N. Wang, and W. Xiang, "Clustering Hierarchy Protocol in Wireless Sensor Networks Using an Improved PSO Algorithm,” IEEE Access, vol. 5, pp. 2241-2253, 2017. https://doi.org/10.1109/ACCESS.2016.2633826

[11] Aripriharta, A. Firmansah, M. Yazid, I. D. Wahyono, Muladi, and G. J. Horng, "Modelling of adaptive power management circuit with feedback for self- powered IoT Modelling of adaptive power management circuit with 
feedback for self-powered IoT,” J. Phys. Conf. Ser., pp. 1-13, 2020. https://doi.org/10.1088/1742$6596 / 1595 / 1 / 012023$

[12] A. Firmansah, Aripriharta, I. M. Wirawan, H. W. Herwanto, I. Fadlika, and Muladi, "Design and Experimental Validation of the Self-powered IoT for Indoor Temperature-Humidity Monitoring," 2019 International Conference on Electrical, Electronics and Information Engineering (ICEEIE), Oct. 2019, pp. 139-143. https://doi.org/10.1109/ICEEIE47180.2019.8981426

[13] A. Trotta, M. di Felice, L. Bononi, E. Natalizio, L. Perilli, E. F. Scarselli, T. S. Cinotti, and R. Canegallo, "BEEDRONES: Energy-efficient Data Collection on Wake-Up Radio-based Wireless Sensor Networks," INFOCOM 2019 - IEEE Conf. Comput. Commun. Work. INFOCOM WKSHPS 2019, pp. 547-553, 2019. https://doi.org/10.1109/INFCOMW.2019.8845046

[14] Muladi, A. Firmansah, Aripriharta, I. A. E. Zaeni, A. N. Handayani, I. M. Wirawan, and G. J. Horng, "Adaptive power management for self-powered IoT on smart shoes," AIP Conf. Proc., vol. 2228, no. April, 2020. https://doi.org/10.1063/5.0001040

[15] N. R. Roy and P. Chandra, "EEDAC-WSN: Energy Efficient Data Aggregation in Clustered WSN," 2019 Int. Conf. Autom. Comput. Technol. Manag. ICACTM 2019, pp. 586-592, 2019. https://doi.org/10.1109/ICACTM.2019.8776679

[16] J. Zhang, P. Hu, F. Xie, J. Long, and A. He, “An Energy Efficient and Reliable In-Network Data Aggregation Scheme for WSN,” IEEE Access, vol. 6, no. c, pp. 71857-71870, 2018. https://doi.org/10.1109/ACCESS.2018.2882210

[17] C. Xu, Z. Xiong, G. Zhao, and S. Yu, "An energy-efficient region source routing protocol for lifetime maximization in WSN," IEEE Access, vol. 7, pp. 135277-135289, 2019. https://doi.org/10.1109/ACCESS.2019.2942321

[18] J. Shen, A. Wang, C. Wang, P. C. K. Hung, and C. F. Lai, "An Efficient Centroid-Based Routing Protocol for Energy Management in WSN-Assisted IoT," IEEE Access, vol. 5, no. 8, pp. 18469-18479, 2017. https://doi.org/10.1109/ACCESS.2017.2749606

[19] S. Khoshabi Nobar, K. Adli Mehr, J. Musevi Niya, and B. Mozaffari Tazehkand, "Cognitive Radio Sensor Network with Green Power Beacon,” IEEE Sens. J., vol. 17, no. 5, pp. 1549-1561, 2017. https://doi.org/10.1109/JSEN.2017.2647878

[20] R. A. Kjellby, L. R. Cenkeramaddi, A. Froytlog, B. B. Lozano, J. Soumya, and M. Bhange, "Long-range Selfpowered IoT Devices for Agriculture Aquaponics Based on Multi-hop Topology," IEEE 5th World Forum Internet Things, WF-IoT 2019 - Conf. Proc., pp. 545-549, 2019. https://doi.org/10.1109/WF-IoT.2019.8767196

[21] D. Ye and M. Z. Au, "A Self-Adaptive Sleep/Wake-Up Scheduling Approach for Wireless Sensor Networks," IEEE Trans. Cybern., vol. 48, no. 3, pp. 979-992, 2018. https://doi.org/10.1109/TCYB.2017.2669996

[22] F. H. Panahi, F. H. Panahi, S. Heshmati, and T. Ohtsuki, "Optimal Sleep Wakeup Mechanism for Green Internet of Things," ICEE 2019 - 27th Iran. Conf. Electr. Eng., pp. 1659-1663, 2019. https://doi.org/10.1109/IranianCEE.2019.8786620

[23] V. L. Quintero, C. Estevez, M. E. Orchard, and A. Pérez, "Improvements of Energy-Efficient Techniques in WSNs: A MAC-Protocol Approach," IEEE Commun. Surv. Tutorials, vol. 21, no. 2, pp. 1188-1208, 2019. https://doi.org/10.1109/COMST.2018.2875810

[24] W. Ye, J. Heidemann, and D. Estrin, “An energy-efficient MAC protocol for wireless sensor networks," Proc. - IEEE INFOCOM, vol. 3, no. October 2014, pp. 1567-1576, 2002. https://doi.org/10.1109/INFCOM.2002.1019408

[25] Z. Tonggang, L. I. U. Kai, Z. Zheng, and P. A. N. Dafa, "Research on the Frequency Hopping Algorithm Based on Long-distance Wireless Sensor Network,” pp. 818-821, 2016. https://doi.org/10.1109/IMCCC.2016.63

[26] T. Wu, "A Distance-Based Scheduling Algorithm With a Proactive Bottleneck Removal Mechanism for Wireless Rechargeable Sensor Networks," vol. 8, 2020. https://doi.org/10.1109/ACCESS.2020.3015911

[27] L. Tang, Z. Lu, and B. Fan, "Energy efficient and reliable routing algorithm for wireless sensors networks," Appl. Sci., vol. 10, no. 5, 2020. https://doi.org/10.3390/app10051885

[28] A. D. Ball, F. Gu, R. Cattley, X. Wang, and X. Tang, "Energy harvesting technologies for achieving self-powered wireless sensor networks in machine condition monitoring: A review," Sensors (Switzerland), vol. 18, no. 12, 2018. https://doi.org/10.3390/s18124113

[29] T. Van Dam and K. Langendoen, "An Adaptive Energy-Efficient MAC Protocol for Wireless Sensor Networks," pp. 171-180, 2003. https://doi.org/10.1145/958491.958512

[30] S.-H. Yang, H.-W. Tseng, E. H. Wu, and G.-H. Chen, "Utilization Based Duty Cycle Tuning MAC Protocol for Wireless Sensor Networks,” pp. 3258-3262, 2005. https://doi.org/10.1109/GLOCOM.2005.1578377

[31] X. Wang, X. Wang, G. Xing, and Y. Yao, "Dynamic Duty Cycle Control for End-to-End Delay Guarantees in Wireless Sensor Networks," IEEE 18th Int. Work. Qual. Serv., 2010. https://doi.org/10.1109/IWQoS.2010.5542743

[32] H. Byun and J. Yu, "Adaptive Duty Cycle Control with Queue Management in Wireless Sensor Networks," vol. 12, no. 6, pp. 1214-1224, 2013. https://doi.org/10.1109/TMC.2012.102

[33] K. Nguyen, V. H. Nguyen, D. D. Le, Y. Ji, D. A. Duong, and S. Yamada, "ERI-MAC: An energy-harvested receiverinitiated MAC protocol for wireless sensor networks," Int. J. Distrib. Sens. Networks, vol. 10, no. 6, 2014. https://doi.org/10.1155/2014/514169

[34] O. Bouachir, A. Ben Mnaouer, F. Touati, and D. Crescini, "EAMP-AIDC - Energy-aware mac protocol with adaptive individual duty cycle for EH-WSN," 2017 13th Int. Wirel. Commun. Mob. Comput. Conf. IWCMC 2017, pp. 20212028, 2017. https://doi.org/10.1109/IWCMC.2017.7986594

[35] Muladi, A. Firmansah, Aripriharta, I. A. E. Zaeni, A. N. Handayani, I. M. Wirawan, and G. J. Horng, "A new battery management system for self-powered smart shoes," AIP Conf. Proc., vol. 2228, no. 1, 2020. 
https://doi.org/10.1063/5.0001041

\section{BIOGRAPHY OF AUTHORS}

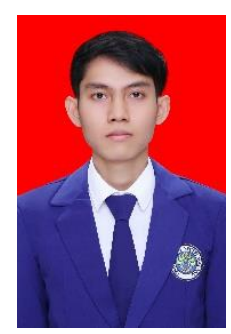

Adim Firmansah received B.Eng. and M.Eng. degrees in Electrical Engineering from Universitas Negeri Malang, Indonesia, in 2019 and 2021. He was active in the research team while taking undergraduate to master's degrees. The focus of his research is wireless sensor networks and intelligent systems. Email: adimfirmansah@gmail.com

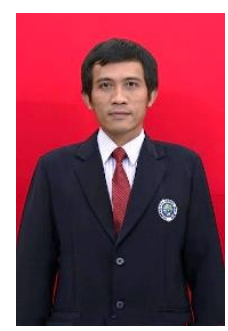

Aripriharta received B.Eng. and M.Eng, a degree in Electrical Engineering from Universitas Brawijaya, Indonesia, in 2002 and 2012, respectively. He received a Ph.D. degree in Electronic Engineering from the National Kaohsiung University of Applied Sciences, Taiwan. Since 2005, he joined the Faculty of Engineering, Universitas Negeri Malang, where he is currently promoted to a professor. His research interests are focused on an intelligent algorithm for wireless sensor networks and its applications on biomedical and smart grids. He is a member of IAENG and IEEE. In 2018, he was a Chair of the Study Program of Electrical Engineering and Chair of Industrial Corporation, Centre of Advanced Material for Renewable Energy, Universitas Negeri Malang. Email: aripriharta.ft@um.ac.id

Sujito received Bachelor (S.T) and Master (M.T) degrees in electrical engineering from

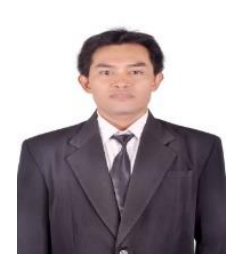
Gadjah Mada University Indonesia in 2000 and 2003, respectively. In 2019, he received Doctor (Ph.D.) degree in electronic engineering from the Institute of Electronic Engineering National Kaohsiung University of Science and Technology Taiwan Republic of China. Since 2003, he joined in electrical engineering, Faculty of Engineering at the State University of Malang (UM). His research interests include power quality, power system control, design of the analog filter, analog signal processing, and analog integrated circuit, and Application of the Internet of Things (IoT). He is a member of IEEE (USA). Email: Sujito.ft@um.ac.id 